\title{
Electronic structure interpolation via atomic orbitals
}

\author{
Mohan Chen, G-C Guo, and Lixin He * \\ Key Laboratory of Quantum Information, University of Science and \\ Technology of China, Hefei, 230026, People's Republic of China
}

(Dated: May 26, 2022)

\begin{abstract}
We present an efficient scheme for accurate electronic structure interpolations based on the systematically improvable optimized atomic orbitals. The atomic orbitals are generated by minimizing the spillage value between the atomic basis calculations and the converged plane wave basis calculations on some coarse $k$-point grid. They are then used to calculate the band structure of the full Brillouin zone using the linear combination of atomic orbitals (LCAO) algorithms. We find that usually $16-25$ orbitals per atom can give an accuracy of about $10 \mathrm{meV}$ compared to the full $a b$ initio calculations. The current scheme has several advantages over the existing interpolation schemes. The scheme is easy to implement and robust which works equally well for metallic systems and systems with complex band structures. Furthermore, the atomic orbitals have much better transferability than the Shirley's basis and Wannier functions, which is very useful for the perturbation calculations.

PACS numbers: 71.15.Ap, 71.15.Dx
\end{abstract}

\section{INTRODUCTION}

Very often one needs to calculate physical properties that need very highly dense $k$ points to get accurate results. For example, Yao et al $\stackrel{\underline{\underline{1}}}{\text { used }} 2 \times 10^{6} k$ points in order to get a converged value of intrinsic anomalous Hall conductivity. Direct calculations of the properties via the first-principles method are too expensive in these cases. An efficient electronic structure interpolation method is therefore a powerful tool to reduce the computational cost while retaining the accuracy at the $a b$ initio level.

Maximally Localized Wannier Functions (MLWFs) 2 have been demonstrated as a powerful tool for interpolating electronic structures accurately ${ }^{3.4}$ It has been used to

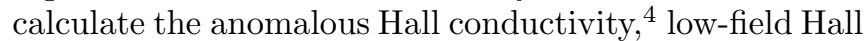
conductivity, and magnetic circular dichroism spectrum,,$\frac{3}{-}$ etc. In this approach, the band connectivity, band crossings and avoided crossings are treated correctly. The evaluation of the velocity matrix can also be performed analytically. Because of their local character, the MLWFs serve as a kind of exact tight-binding basis of minimal sizes. However, in practice, it is not always easy to generate highly localized Wannier functions for metallic systems, and systems with complex band structures.

The electronic structures can also be interpolated by the Bloch states. Shirley proposed an interpolation scheme in $1996 . \underline{\underline{5}}$ The optimal basis set is extracted from the full $a b$ initio calculations on a coarse $k$-point grid. It has been shown that by using about 35 basis functions per atom, the optimal basis can reproduce the band structures of $\mathrm{Si}$ and LiF within an accuracy of $10 \mathrm{meV}$. Recently, Shirley's scheme has been extended to more general $k$-point sampling. $\underline{\underline{6}}$ The Shirley's method can be used well for both insulators and metals. However, the

\footnotetext{
*corresponding author: helx@ustc.edu.cn
}

Shirley's basis is delocalized, therefore it can not take the advantages of the locality as MLWFs. Furthermore, the Shirley's scheme requires storing a large number of wave functions, which might be problematic for large systems.

Atomic orbitals have been popular recently for selfconsistent $a b$ initio calculations. Relatively compact basis size can give quite satisfactory results compared to the plane wave calculations ${ }^{7-10}$ However, to get extremely high accuracy band structures (errors $\sim 2-3 \mathrm{meV}$ ), the small, rigid atomic basis sets are obviously not adequate. Recently the authors (CGH) developed a method to generate systematically improvable fully optimized atomic orbitals for $a b$ initio calculations. 11 The CGH scheme can generate orbitals with arbitrary angular momentum and multi-zeta functions in a unified procedure. The CGH orbitals offer highly accurate and transferable atomic basis sets, which have been tested for a wide variety of systems, including semiconductors, oxides, metals, clusters, etc.

We show in this paper that the CGH method can be revised to give an efficient scheme for electronic structures interpolations. This scheme keeps the advantages of both MLWFs and Shirley's methods and gets rid of some of their disadvantages. The atomic orbitals are highly localized (more localized than MLWFs), and therefore can take the advantage of the locality. The atomic orbitals are very neat, and only the one dimensional radial functions are stored. We show that the scheme works well for both metals and insulators. The highly localized orbitals can always be generated for metallic systems. Additionally, the transferbility of local orbitals for interpolation purposes is explored. Once the atomic orbitals are generated, the electronic properties can be calculated efficiently by the well established linear combination of atomic orbitals (LCAO) algorithms, with the accuracy of $a b$ initio plane wave calculations.

The rest of the paper is organized as follows. In Sec. III we introduce briefly the method we use to do the band 
structure interpolation via atomic orbitals. In Sec. III, we show, via a few examples, how the present interpolation scheme works for both metallic and insulating systems, and systems with complex band structures. We also discuss the transferability of our interpolation bases. We conclude in Sec. [IV]

\section{METHODS}

All the calculations performed in this paper are based on the density functional theory (DFT) within local (spin) density approximation $[\mathrm{L}(\mathrm{S}) \mathrm{DA}]$. Although our method is demonstrated for the norm-conserving 12 pseudopotentials, in principle, it should also work well for the ultrasoft or PAW pseudopotentials. Monkhorst-Pack $k$ points 13 are used in the following calculation.

\section{A. Generating local orbitals}

The details of how to construct systematically optimized atomic basis for ab initio calculations have been given in Ref. 11. Here we give a brief review of our earlier approach to generating local orbitals. The atomic orbitals are written as radial functions times spherical harmonic functions, i.e., $\phi_{\mu}(\mathbf{r})=f_{\mu, l}(r) Y_{l m}(\hat{r})$. The radial function is taken as the linear combination of Spherical Bessel functions within certain range $r_{c}$, i.e.,

$$
f_{\mu, l}(r)= \begin{cases}\sum_{q} c_{\mu q} j_{l}(q r), & r<r_{c} \\ 0 & r \geq r_{c} .\end{cases}
$$

where $\mu=\{\alpha, i, \zeta, l, m\}$, and $\alpha, i, \zeta, l, m$ are the element type, the index of atom of each element type, the multiplicity of the radial functions, the angular momentum and the magnetic quantum number, respectively. $j_{l}(q r)$ is the spherical Bessel function, where $q$ satisfy $j_{l}\left(q r_{c}\right)=0$. The coefficients $c_{\mu q}$ are chosen to minimize the "spillage",

$$
\mathcal{S}=\frac{1}{N_{n} N_{k}} \sum_{n=1}^{N_{n} N_{k}}\left\langle\Psi_{n}(\mathbf{k})|1-\hat{P}(\mathbf{k})| \Psi_{n}(\mathbf{k})\right\rangle,
$$

between the Hilbert space spanned by the atomic orbitals and the plane wave calculations, for selected reference states. In Eq. 2, $\Psi_{n}(\mathbf{k})$ is the eigenstate of plane wave calculations, and $N_{n}$ and $N_{k}$ are the number of states of interest and the number of k-points in the Brillouin zone. $\hat{P}(\mathbf{k})$ is a projector spanned by the atomic orbitals, i.e.,

$$
\hat{P}(\mathbf{k})=\sum_{\mu \nu}\left|\phi_{\mu}(\mathbf{k})\right\rangle S_{\mu \nu}^{-1}(\mathbf{k})\left\langle\phi_{\nu}(\mathbf{k})\right|,
$$

with

$$
\left\langle\mathbf{r} \mid \phi_{\mu}(\mathbf{k})\right\rangle=\sum_{\mathbf{R}} \phi_{\mu}\left(\mathbf{r}-\mathbf{r}_{\mu}-\mathbf{R}\right) e^{i \mathbf{k} \cdot\left(\mathbf{r}_{\mu}+\mathbf{R}\right)},
$$

where $\phi_{\mu}(\mathbf{r})$ is atomic orbitals. $\mathbf{r}_{\mu}$ is the atom center of the $\mu$-th orbital, and $\mathbf{R}$ is the lattice vector. $S_{\mu \nu}(\mathbf{k})=\left\langle\phi_{\mu}(\mathbf{k}) \mid \phi_{\nu}(\mathbf{k})\right\rangle$ is the overlap matrix element. The minimization of the spillage is achieved by using a simulated annealing method. Because the orbitals generated from this step usually have unphysical oscillations, an additional step is added to smooth out the orbitals by minimizing the kinetic energy of each orbital 11

We have performed extensive tests of this scheme for a wide variety of systems, including semiconductors, oxides, metals, and clusters, etc. The results show that the obtained atomic bases are very satisfactory in both accuracy and transferability: $\underline{11}$ However, to make high accuracy electronic structure interpolation, we need to modify the scheme for the task.

In Ref. 11, where the aim is to do self-consistent $a b$ initio calculations in complex chemical environments, to get maximal transferability of the basis sets, a set of dimers with different bond lengths are selected as the reference systems. The valence states are chosen as the reference states. However, in order to interpolate the electronic structures with extremely high accuracy for a particular system, the system itself is used as the reference system. In many cases, the interested states lie only in a given energy window (i.e., valence bands as well as conduction bands), therefore, we can choose these states (instead of all the valence bands) as the references states.

In the self-consistent LCAO calculations, which are usually applied to deal with extremely large systems, it is important to keep the basis size compact. Often used basis are DZP (double zeta orbitals plus a polar orbital) etc. The small basis set is not enough for high accuracy electronic structure interpolations. One must use much higher angular momentum and more zeta orbitals. In these cases, the CGH scheme has obvious advantages, because it can generate the fully optimized orbitals with arbitrary angular momentum and any number of radial functions for a given angular momentum.

It has been shown in Ref. 11 that increasing the orbital radius cutoff $r_{c}$ can reduce the spillage value, and therefore improve the quality of the orbitals. However, a larger $r_{c}$ also means more computational costs. In the self-consistent calculations, one has to balance the computational costs and the accuracy, and choose a modest $r_{c}$. Yet, for band structure interpolations, the systems under study are usually considerably smaller, a larger $r_{c}$ can be used.

Once we obtain the atomic orbitals, the electronic structures can be calculated efficiently by using well established LCAO methods.

\section{RESULTS AND DISCUSSIONS}

In this Section, we show via a few examples how the present band structure interpolation scheme works. We start the tests from simple solid structures like $\mathrm{Na}, \mathrm{Si}$ and $\mathrm{Al}$. $\mathrm{Na}$ and $\mathrm{Al}$ are metallic while $\mathrm{Si}$ is a semiconductor. 

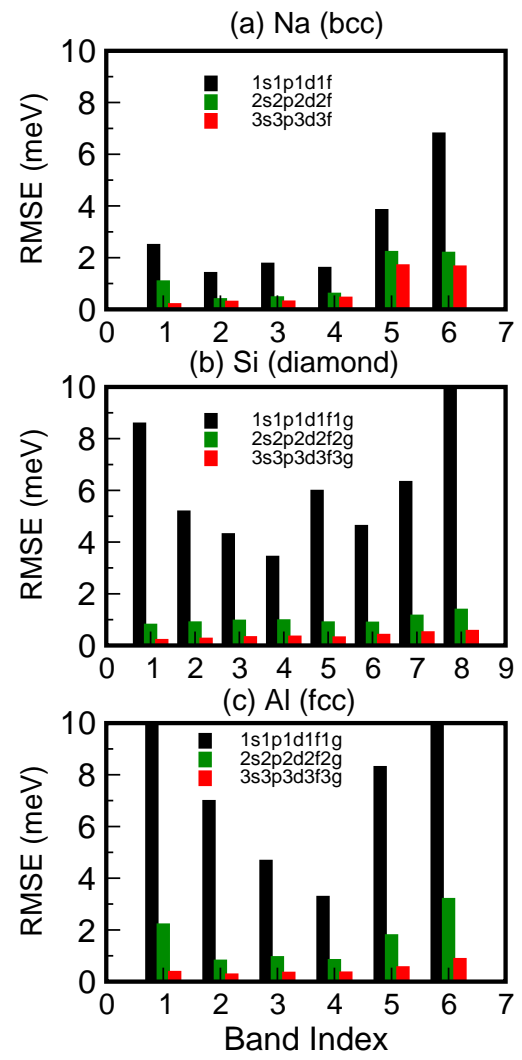

FIG. 1: (Color online) The RMSE of interpolated bands of (a) bcc Na; (b) Si diamond structure; (c) fcc Al. The black, red, and green columns represent the RMSE of Level 1, 2, 3 atomic basis sets respectively.

More complex materials, such as iron based superconductor $\mathrm{BaFe}_{2} \mathrm{As}_{2}$ are then tested. We further show that the atomic bases have much better transferability than the the Bloch bases and Wannier bases for the purpose of band structure interpolation.

\section{A. Simple Solids}

We take bcc Sodium as the first example. We use energy cut-off $E_{c}=50 \mathrm{Ry}$. A $8 \times 8 \times 8 k$-point mesh is used as the reference states to generate the local orbitals. We choose the lowest 6 bands for interpolation. In order to quantify the quality of the interpolation, we calculate the root-mean-square errors (RMSE) between the interpolated bands and the plane wave calculations, defined as,

$$
\mathrm{RMSE}=\sqrt{\sum_{i=1}^{N}\left(\epsilon_{i}^{\mathrm{PW}}-\epsilon_{i}^{\mathrm{LCAO}}\right)^{2} / N}
$$

where $N$ is the number of the $k$ points that the comparison has been taken between $\epsilon^{\mathrm{PW}}$ the eigenvalue calculated from plane wave basis and $\epsilon^{\mathrm{LCAO}}$ the eigenvalue calculated from atomic orbitals.

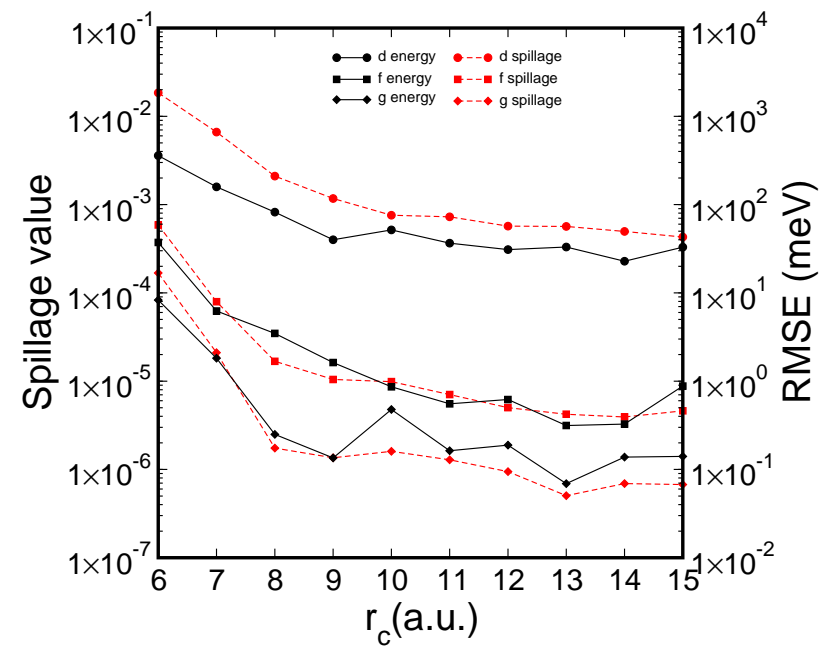

FIG. 2: (Color online) The spillage values and the averaged RMSE as functions of radial cutoff $r_{c}$ for the basis sets, whose maximal angular momentum are d, f, g, respectively.

The RMSE of the 6 interpolated bands of Na are shown in Fig.11(a). Three levels of atomic orbitals are generated and compared. At each level, we use one group of s, p, $\mathrm{d}$, and f orbitals $(1 \mathrm{~s} 1 \mathrm{p} 1 \mathrm{~d} 1 \mathrm{f})$. The radius cutoff $r_{c}$ is chosen as 12 a.u. We find that the quality of the basis set is much better if all the orbitals of the same level are generated simultaneously than those generated separately. As shown in Fig. 1(a), the RMSE of the 1s1p1d1f, 2s2p2f2f and $3 \mathrm{~s} 3 \mathrm{p} 3 \mathrm{~d} 3 \mathrm{f}$ local basis sets are less than $8 \mathrm{meV}, 3 \mathrm{meV}$ and $2 \mathrm{meV}$ respectively.

Figure 2 illustrates how the angular momentums and radial cutoff affect the interpolation accuracy. The black lines indicate the spillage values while the red lines indicate the averaged RMSE of the 6 bands. The radius cutoff changes from 6 a.u. to 15 a.u.. The largest angular momentum of the orbitals is set from $l=2$ (d orbitals) to $l=4$ (g orbitals). We use 5 radial orbitals (zeta functions) for each angular momentum, which ensures that the accuracy improved by multi-zeta orbitals is converged. We find consistent change between spillage values and the RMSE, confirming that the spillage is a good criterion for the interpolation quality. The RMSE reduce considerably as $r_{c}$ increases from 6 a.u. to 9 a.u., while only small improvement is found when $r_{c}$ is larger than 9 a.u.. The d orbitals are not sufficient for the high accuracy interpolation, because the corresponding RMSE are around 50 to $100 \mathrm{meV}$ even a very large $r_{c}$ is used. Adding in f orbitals improve greatly upon d orbitals. For example, for $r_{c}=10$ a.u., adding f orbitals to the interpolation basis reduces the RMSE to $0.86 \mathrm{meV}$. The $g$ orbitals improve further upon f orbitals, but not as much as f orbitals to d orbitals. Since the spillage can be as small as $10^{-6}$, one can get highly accurate physical properties related to the wave functions.

The interpolation results for Si diamond structure and fcc $\mathrm{Al}$ are shown in Fig. 1(b)(c) respectively. For these 

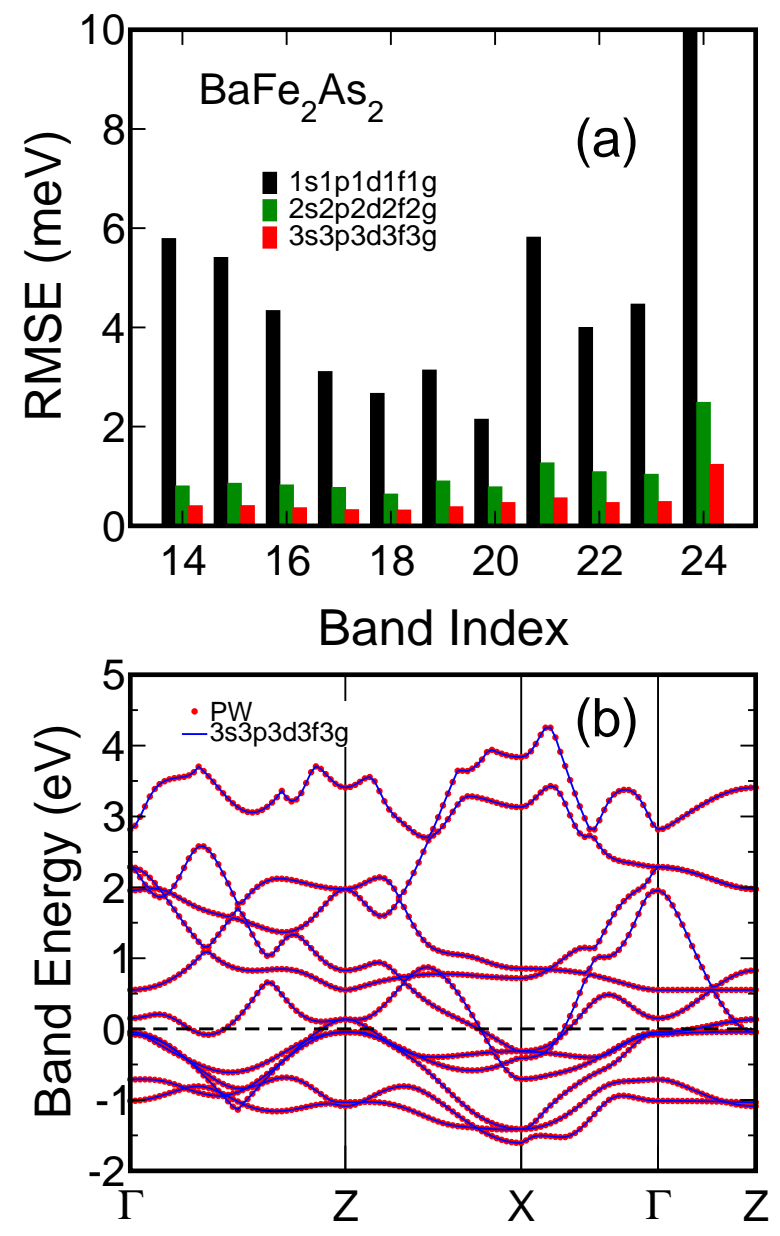

FIG. 3: (Color online) (a) The RMSE of interpolated bands for $\mathrm{BaFe}_{2} \mathrm{As}_{2}$. The black, red, and green columns represent the RMSE of Level 1, 2, 3 atomic basis sets respectively. (b) Compare the interpolated band structures (blue lines) using 3s3p3d3f3g basis to the plane wave calculations (red dots).

two materials, we find $\mathrm{g}$ orbitals must be included to get highly accurate interpolation results. For Si, the lowest 8 bands are interpolated. The 1s1p1d1f1g orbitals can interpolate the band structures with RMSE are about 10 $\mathrm{meV}$ for all the bands. If the basis set is doubled, the RMSE for all 8 bands are smaller than $2 \mathrm{meV}$, showing extremely good accuracy. When it further increases to the level 3 orbitals, the RMSE can be reduced within 1 $\mathrm{meV}$. For $\mathrm{Al}$, if the first 1s1p1d1f1g orbitals are used, the RMSE can be reduced to $10 \mathrm{meV}$. When we double the basis to 2s2p2d2f2g, and RMSE of all the bands fall within $3 \mathrm{meV}$. The RMSE can be further reduced to less than $1 \mathrm{meV}$, when 3s3p3d3f3g orbitals are used, also showing remarkable accuracy.

\section{B. $\mathrm{BaFe}_{2} \mathrm{As}_{2}$}

The recent discovery of superconductivity in doped iron arsenide has attracted great attention $\underline{16}$ Here we show our electronic structure interpolation scheme also works well for materials like $\mathrm{BaFe}_{2} \mathrm{As}_{2}$ which have complex band structures. We interpolate the 14th to 24th bands, which span about a $6 \mathrm{eV}$ energy window around the Fermi level. Figure 3(a) depicts the RMSE for each band using different numbers of numerical orbitals. When using the 1s1p1d1f1g orbital (25 basis per atom), the RMSE of all the bands are under $6 \mathrm{meV}$ except for the last one, which is $15.7 \mathrm{meV}$. Once the second radial functions are used, the RMSE of all bands are reduced to below $1.3 \mathrm{meV}$ except the last one which is $2.48 \mathrm{meV}$. When using the third radial functions, all the RMSE are under $0.6 \mathrm{meV}$ except the last one which is $1.23 \mathrm{meV}$.

Figure 3(b) depicts the band structures of $\mathrm{BaFe}_{2} \mathrm{As}_{2}$ along the $\Gamma-\mathrm{Z}-\mathrm{X}-\Gamma-\mathrm{Z}$ line. The band structures calculated by plane waves are shown in blue dotted line,

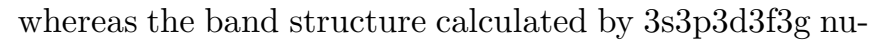
merical orbitals are plotted in black solid lines. The interpolated bands and the full $a b$ initio calculations are essentially indistinguishable.

We also test GaAs, fcc $\mathrm{Cu}$ and $\mathrm{CuZn}$ alloys, and the results are all similar to the the examples above. For GaAs and fcc $\mathrm{Cu}$, the RMSE are less than $10 \mathrm{meV}$ by using the 1s1p1d1f1g orbitals (25 atomic orbitals per atom) only. We find that the 3s3p3d3f orbitals (48 orbitals per atom) can interpolate the 60 bands around fermi level of $\mathrm{CuZn}$ alloys with RMSE $\sim 2 \mathrm{meV}$, which are quite similar to the results given in Ref. 6, in which 42 bases per atom were used to get the same accuracy $\underline{\underline{6}}$

\section{TRANSFERBILITY}

Sometimes we need to calculate the physical properties under certain perturbations, such as strain, defects, electric field, etc. In these cases, a transferable basis set is highly desirable. Shirley's Bloch interpolation does not have any transferbility. Even with a small change of the system (e.g., a deformation of the unit cell), the Bloch basis needs to be regenerated. It has been shown that Wanner functions do not have satisfactory transferbility either, $\stackrel{17,18}{18}$ because they have too many details. Here we demonstrate that the CGH orbitals have very good transferbility for the interpolation purpose.

We take the $(3,3)$ carbon nanotube $(\mathrm{CNT})$ as an example, which is a metallic armchair nanotube. The system contains 12 atoms. We interpolate the 20th to 27 th bands around the Fermi level. We use the energy cutoff $E_{c}=100$ Ry. We first generate the interpolation basis for the CNT at its equilibrium structure. We use s, p, d and $\mathrm{f}$ orbitals as the interpolation basis, with the radius cutoff $r_{c}=10$ a.u. The RMSE are shown in Fig. 4(a). The conduction bands are more difficult to interpolate than valence bands, but still for Level 2, 3, 4 orbitals, the RMSE are less than $8 \mathrm{meV}, 4 \mathrm{meV}$ and $2 \mathrm{meV}$ respectively. The interpolated band structures are shown in Fig. 4(d) in black solid lines compared to the plane wave results shown in the red dotted lines. The two band structures are hardly 

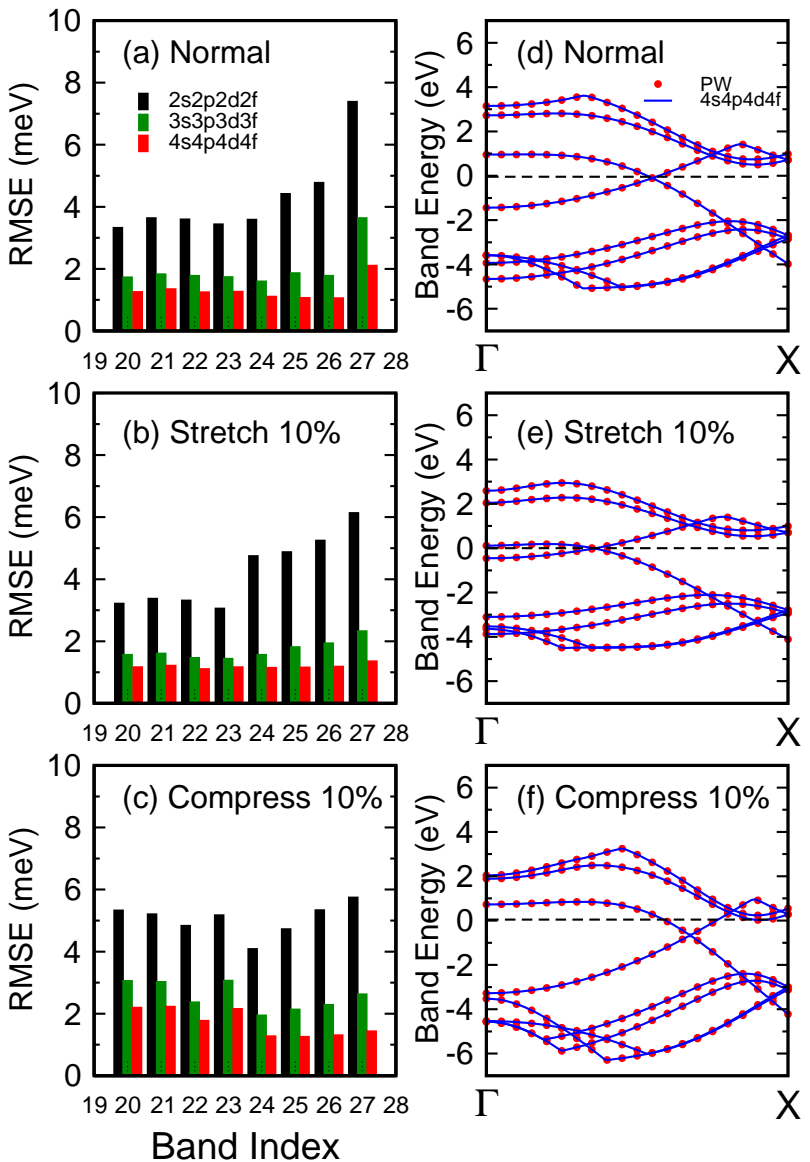

FIG. 4: (Color online) The RMSE of the interpolated bands for the $(3,3)$ carbon nanotube of (a) normal, (b) with $10 \%$ stretching, and (c) with $10 \%$ compression, structures. (d), (e), (f) show corresponding interpolated band structures (blue lines) using $4 \mathrm{~s} 4 \mathrm{p} 4 \mathrm{~d} 4 \mathrm{f}$ basis compared to the plane wave results (red dots).

distinguishable.

Using the same interpolation basis, we calculate the band structures for $(3,3)$ CNTs which are stretched $10 \%$ or compressed $10 \%$ along the tube and their RMSE. The RMSE are given in Fig. 4(b)(c), and the corresponding band structures are shown in Fig. 4 (e)(f) respectively. As we see, the RMSE almost do not change with respect to the CNT stretching and compressing, even though the band structures themselves change dramatically. These results demonstrate that the $\mathrm{CGH}$ orbitals have excellent transferbility for the interpolation purpose, which is very important when perturbation calculations are needed.

\section{CONCLUSION}

We have presented an efficient scheme for accurate electronic structure interpolations based on the systematically improvable optimized atomic orbitals. The current scheme has several advantages over the existing interpolation schemes. We find usually $16-25$ orbitals per atom can give accuracy about $10 \mathrm{meV}$ compared to the full $a b$ initio calculations, similar to that of the revised Shirley's scheme. However, unlike the Shirley's method, in which a large number of three-dimensional wave functions have to be stored in the calculations, the present scheme only needs to store the one-dimensional radial functions. The atomic orbitals are highly localized, therefore the scheme has many good features as the interpolation schemes based on maximally localized Wannier functions. Even though the number of atomic orbitals is greater than that of Wannier functions, generally the atomic orbitals are more localized than Wannier functions, and therefore have much less neighboring atoms. The scheme is easy to implement and robust, working equally well for metallic systems and systems with complex band structures. Furthermore, the atomic orbitals have much better transferability than the Shirley's basis and Wannier functions, which is very useful for the perturbation calculations.

\section{Acknowledgments}

LH acknowledges support from the Chinese National Fundamental Research Program 2011CB921200, "Hundreds of Talents" program from the Chinese Academy of Sciences and National Natural Science Funds for Distinguished Young Scholars.
${ }^{1}$ Y. Yao, L. Kleinman, A. H. MacDonald, J. Sinova, T. Jungwirth, D. S. Wang, E. Wang, and Q. Niu, Phys. Rev. Lett 92, 037204 (2004).

2 N. Marzari and D. Vanderbilt, Phys. Rev. B 56, 12847 (1997).

3 X. Wang, J. R. Yates, I. Souza, and D. Vanderbilt, Phys. Rev. B 74, 195118 (2006).

4 J. R. Yates, X. Wang, D. Vanderbilt, and I. Souza, Phys. Rev. B 75, 195121 (2007).

5 E. L. Shirley, Phys. Rev. B 54, 16464 (1996).

6 D. Prendergast and S. G. Louie, Phys. Rev. B 80, 235126 (2009).
7 S. D. Kenny, A. P. Horsfield, and H. Fujitani, Phys. Rev. B 62, 4899 (2000).

8 J. Junquera, O. Paz, D. Sanchez-Portal, and E. Artacho, Phys. Rev. B 64, 235111 (2001).

9 J. M. Soler, E. Artacho, J. D. Gale, A. Garcia, J. Junquera, P. Ordejon, and D. S. Portal, J. Phys:: Condenss. Matter 14, 2745 (2002).

10 T. Ozaki, Phys. Rev. B 67, 155108 (2003).

11 M. Chen, G. C. Guo, and L. He, J. Phys.:Condens. Matter 22, 445501 (2010).

12 D. R. Hamann, M. Schluter, and C. Chiang, Phys. Rev. Lett 43, 1494 (1979). 
13 H. J. Monkhorst and J. D. Pack, Phys. Rev. B 13, 5188 (1976).

14 D. S. Portal, E. Artacho, and J. M. Soler, Solid State Communications 95, 685 (1995).

15 D. S. Portal, E. Artacho, and J. M. Soler, J. Phys: Condes Matter 8, 3859 (1996).

16 Y. Kamihara, H. Hiramatsu, M. Hirano, R. Kawamura,
H. Yanagi, T. Kamiya, and H. Hosono, J. Am. Chem. Soc. 128, 10012 (2008).

17 W. Hierse and E. B. Stechel, Phys. Rev. B 54, 16515 (1996).

18 M. Chen, W. Fang, G. Z. Sun, G. C. Guo, and L. He, Phys. Rev. B 80, 165121 (2009). 